\title{
Upper semicontinuity of attractors for small perturbations of Klein-Gordon-Schrödinger lattice system
}

Hengyan $\mathrm{Li}^{1 *}$ and Lei Sun ${ }^{1,2}$

"Correspondence:

hengyanli989@126.com

1school of Mathematics and

Information Sciences, North China University of Water Resources and

Electric Power, Zhengkai Road,

Zhengzhou, 450011, P.R. China

Full list of author information is

available at the end of the article

\begin{abstract}
This paper studies the existence of the random attractor for a Klein-Gordon-Schödinger system under a small $\varepsilon$-random perturbation on a high dimensional infinite lattice. Firstly, we prove the asymptotic compactness of the random dynamical system and obtain the random attractor. Then, by comparing to the case without random perturbation $(\varepsilon=0)$, we show the upper semicontinuity of the attractors.
\end{abstract}

Keywords: random attractor; stochastic Klein-Gordon-Schrödinger system; upper semicontinuity

\section{Introduction}

In this paper, we consider the discrete Klein-Gordon-Schrödinger system with a small random perturbation,

$$
\begin{aligned}
& i\left(\frac{d u_{j}}{d t}+\alpha u_{j}\right)-(A u)_{j}+u_{j} v_{j}=f_{j}(t)+\varepsilon a_{j} u_{j} \frac{d w_{j}^{1}}{d t}, \\
& \frac{d^{2} v_{j}}{d t^{2}}+(A v)_{j}+\mu \frac{d v_{j}}{d t}+v_{j}-\left|u_{j}\right|^{2}=g_{j}(t)+\varepsilon b_{j} \frac{d w_{j}^{2}}{d t},
\end{aligned}
$$

with initial conditions

$$
u_{j}(0)=u_{j 0}, \quad v_{j}(0)=v_{j 0}, \quad \dot{v}_{j}(0)=v_{1 j 0},
$$

where $j \in \mathbb{Z}^{n}, n \leq 3, t>0, \mathbb{Z}$ is the integer set, $\alpha, \mu$, and $\varepsilon$ are positive constants, $i^{2}=-1$, $a=\left(a_{j}\right)_{j \in \mathbb{Z}^{n}} \in \ell^{2}$, and $b=\left(b_{j}\right)_{j \in \mathbb{Z}^{n}} \in \ell^{2}, f(t)=\left(f_{j}(t)\right)_{j \in \mathbb{Z}^{n}}, g(t)=\left(g_{j}(t)\right)_{j \in \mathbb{Z}^{n}} \in \mathbb{C}_{b}\left(\mathbb{R}, \ell^{2}\right)$, the space of bounded continuous functions from $\mathbb{R}$ into $\ell^{2} .\left\{w_{j}^{1}(t): j \in \mathbb{Z}^{n}\right\}$ and $\left\{w_{j}^{2}(t): j \in \mathbb{Z}^{n}\right\}$ are two independent two-side real valued standard Wiener processes, linear operator $A$ and space $\ell^{2}$ will be described in detail in the next section.

The coupled Klein-Gordon-Schrödinger (KGS) system is an important model in nonlinear science. It is encountered in several diverse branch of physics, for example in the description of the interaction of a scalar nucleon interacting with a neutral scalar meson,

@2014 Li and Sun; licensee Springer. This is an Open Access article distributed under the terms of the Creative Commons Attribution License (http://creativecommons.org/licenses/by/2.0), which permits unrestricted use, distribution, and reproduction in any medium, provided the original work is properly cited. 
as in the following nonautonomous KGS equations in $\mathbb{R}$ :

$$
\begin{aligned}
& i\left(u_{t}+\alpha u\right)+\Delta u+u v=f(t, x), \\
& v_{t t}-\Delta v+v v_{t}+\mu v-\beta|u|^{2}=g(t, x),
\end{aligned}
$$

where $u$ denotes a complex scalar nucleon field and $v$ represents a real meson field; the complex-valued function $f(t, x)$ and the real-valued function $g(x, t)$ both are the timedependent external sources. By using the Galerkin method, Fukuda and Tsutsumi [1] first studied the coupled KGS equations, and they obtained the existence of global strong solutions. We refer the readers to [2-7] for the existence of global solutions, asymptotic behavior, and stability for the KGS system and KGS system on infinite lattices.

Various properties of solutions for lattice dynamical systems (LDSs) have been extensively investigated. For example, the long time behavior of LDSs were studied in [6, 8-17]; the traveling wave solutions of LDSs were studied in [18]; the chaotic properties of solutions for LDSs were examined in [11]. Lattice dynamical systems play an important role in their potential applications such as biology [19], chemical reactions [20], pattern recognition and image processing [21], electrical engineering [22], laser systems [23], etc. However, a system in reality is usually affected by external perturbations, which in many cases are of great uncertainty or have a random influence. These random effects are not only introduced to compensate for the defects in some deterministic models but also to explain the intrinsic phenomena. Therefore, there is much work concerned with stochastic lattice dynamical systems [24-27]. To the authors' knowledge, Ruelle [28] first introduced a corresponding generalization of the attractor (random attractor) to the stochastic PDEs. After that, the study of random attractors gained considerable attention during the past decade; see [29, 30] for a comprehensive survey. Bates et al. [24] first investigated the existence of global random attractor for a kind of first order dynamical systems driven by white noise on lattice $\mathbb{Z}$; then Lv and Sun [25] extended the results of Bates to the higher dimensional lattices. After that, there are several papers considering with stochastic evolution equations in an infinite lattice [26, 27, 31]. In this paper, we first extend the result [31] to the higher dimensional lattices, then, by comparing to the case without random perturbation [16] $(\varepsilon=0)$, we see the relationship between a random attractor and a global attractor for a small $\varepsilon$ random perturbed Klein-Gordon-Schrödinger lattice system, i.e. upper semicontinuity of the attractors for a small $\varepsilon$ perturbed Klein-Gordon-Schrödinger lattice system. Roughly speaking, let $A_{\varepsilon}$ be the attractor of the perturbed system, $A_{0}$ be the attractor of the unperturbed system; we say that those attractors have upper semicontinuity if

$$
\lim _{\varepsilon \rightarrow 0} \operatorname{dist}\left(A_{\varepsilon}, A_{0}\right)=0
$$

where dist $(\cdot, \cdot)$ denotes the Hausdorff semidistance.

This paper is organized as follows. In Section 2, we recall some basic concepts and already known results related to random dynamical systems and random attractors. In Section 3, we prove the existence of the random attractor for stochastic KGS lattice dynamical systems of (1.1) on $\mathbb{Z}^{n}$. In Section 4, by comparing to the case without the random perturbation, i.e. $\varepsilon=0$ case, we obtain the upper semicontinuity of the attractors. 


\section{Preliminaries}

In this section, we first introduce the definitions of the random dynamical systems and random attractor, which are taken from $[24,30]$.

Let $\left(H,\|\cdot\|_{H}\right)$ be a Hilbert space, $(\Omega, \mathbb{F}, \mathbb{P})$ be a probability space.

Definition $\left(\Omega, \mathbb{F}, \mathbb{P},\left(\theta_{t}\right)_{t \in \mathbb{R}}\right)$ is called a metric dynamical systems, if $\theta: \mathbb{R} \times \Omega \rightarrow \Omega$ is $(\mathbb{B}(\mathbb{R}) \times \mathbb{F}, \mathbb{F})$-measurable, $\theta_{0}=\mathbb{I}, \theta_{t+s}=\theta_{t} \circ \theta_{s}$ for all $t, s \in \mathbb{R}$, and $\theta_{t} \mathbb{P}=\mathbb{P}$ for all $t \in \mathbb{R}$.

Definition A stochastic process $\phi(t, \omega)$ is called a continuous random dynamical system (RDS) over $\left(\Omega, \mathbb{F}, \mathbb{P},\left(\theta_{t}\right)_{t \in \mathbb{R}}\right)$ if $\phi$ is $\left(\mathbb{B}\left(\mathbb{R}^{+}\right) \times \mathbb{F} \times \mathbb{B}(H), \mathbb{B}(H)\right)$-measurable, and for all $\omega \in \Omega$

- the mapping $\phi: \mathbb{R}^{+} \times \Omega \times H \rightarrow H$ is continuous;

- $\phi(0, \omega)=\mathbb{I}$ on $H$

- $\phi(t+s, \omega, x)=\phi\left(t, \theta_{s} \omega, \phi(s, \omega, x)\right)$ for all $t, s \geq 0$ and $x \in H$ (cocycle property).

Definition 2.1 A random bounded set $B(\omega) \subset H$ is called tempered with respect to $\left(\theta_{t}\right)_{t \in \mathbb{R}}$ if for a.e. $\omega \in \Omega$ and all $\varepsilon>0$

$$
\lim _{t \rightarrow \infty} e^{-\varepsilon t} d\left(B\left(\theta_{-t} \omega\right)\right)=0,
$$

where $d(B)=\sup _{x \in B}\|x\|_{H}$.

Consider a continuous random dynamical system $\phi(t, \omega)$ over $\left(\Omega, \mathbb{F}, \mathbb{P},\left(\theta_{t}\right)_{t \in \mathbb{R}}\right)$ and let $\mathbb{D}$ be the collection of all tempered random set of $H$.

Definition 2.2 A random set $\mathbb{K}(\omega)$ is called an absorbing set in $\mathbb{D}$ if for all $B \in \mathbb{D}$ and a.e. $\omega \in \Omega$ there exists $t_{B}(\omega)>0$ such that

$$
\phi\left(t, \theta_{-t} \omega, B\left(\theta_{-t} \omega\right)\right) \subset \mathbb{K}(\omega), \quad t \geq t_{B}(\omega)
$$

Definition 2.3 A random set $\mathbb{A}(\omega)$ is a random $\mathbb{D}$-attractor for RDS $\phi$ if

- $\mathbb{A}(\omega)$ is a random compact set, i.e., $\omega \rightarrow d(x, \mathbb{A}(\omega))$ is measurable for every $x \in H$ and $\mathbb{A}(\omega)$ is compact for a.e. $\omega \in \Omega$;

- $\mathbb{A}(\omega)$ is strictly invariant, i.e., $\phi(t, \omega, \mathbb{A}(\omega))=\mathbb{A}\left(\theta_{t} \omega\right), \forall t \geq 0$ and for a.e. $\omega \in \Omega$;

- $\mathbb{A}(\omega)$ attracts all sets in $\mathbb{D}$, i.e., for all $B \in \mathbb{D}$ and a.e. $\omega \in \Omega$ we have

$$
\lim _{t \rightarrow \infty} d\left(\phi\left(t, \theta_{-t} \omega, B\left(\theta_{-t} \omega\right)\right), \mathbb{A}(\omega)\right)=0
$$

where $d(X, Y)=\sup _{x \in X} \inf _{y \in Y}\|x-y\|_{H}, X, Y \subset H$.

The collection $\mathbb{D}$ is called the domain of attraction of $\mathbb{A}$.

Definition 2.4 Let $\phi$ be a RDS on Hilbert space $H . \phi$ is called asymptotically compact if for any bounded sequence $\left\{x_{n}\right\} \subset H$ and $t_{n} \rightarrow \infty$, the set $\left\{\phi\left(t_{n}, \theta_{-t_{n}} \omega, x_{n}\right)\right\}$ is precompact in $H$, for any $\omega \in \Omega$.

From [24], we have the following result. 
Proposition 2.1 Let $\mathbb{K} \in \mathbb{D}$ be an absorbing set for an asymptotically compact continuous $R D S \phi$. Then $\phi$ has a unique global random $\mathbb{D}$-attractor

$$
\mathbb{A}(\omega)=\bigcap_{\kappa \geq t_{\mathbb{K}(\omega)}} \overline{\bigcup_{t \geq \kappa} \phi\left(t, \theta_{-t} \omega, \mathbb{K}\left(\theta_{-t} \omega\right)\right)}
$$

which is compact in $H$.

Throughout this paper, let $n \in \mathbb{N}$ be a fixed positive integer. We set

$$
\begin{aligned}
& \mathbb{L}^{2}=\left\{u=\left.\left(u_{j}\right)_{j \in \mathbb{Z}^{n}}\left|j=\left(j_{1}, j_{2}, \ldots, j_{n}\right) \in \mathbb{Z}^{n}, u_{j} \in \mathbb{C}: \sum_{j \in \mathbb{Z}^{n}}\right| u_{j}\right|^{2}<\infty\right\}, \\
& \ell^{2}=\left\{v=\left(v_{j}\right)_{j \in \mathbb{Z}^{n}} \mid j=\left(j_{1}, j_{2}, \ldots, j_{n}\right) \in \mathbb{Z}^{n}, v_{j} \in \mathbb{R}: \sum_{j \in \mathbb{Z}^{n}} v_{j}^{2}<\infty\right\} .
\end{aligned}
$$

For brevity, we use $H$ to denote the Hilbert space $\mathbb{L}^{2}$ or $\ell^{2}$, and we equip $H$ with the inner product and norm as

$$
(u, v)=\operatorname{Re} \sum_{j \in \mathbb{Z}^{n}} u_{j} \bar{v}_{j}, \quad\|u\|^{2}=(u, u)=\sum_{j \in \mathbb{Z}^{n}}|u|^{2}, \quad \forall u, v \in H
$$

where $\bar{v}_{j}$ denotes the conjugate of $v_{j}$.

In our paper, we introduce the transformation $\psi=\frac{d v_{j}}{d t}+\sigma v_{j}$ with $\sigma=\frac{\mu}{\mu^{2}+4}<\min \{\mu, 1\}$ a small positive constant. Then system (1.1) becomes

$$
\begin{aligned}
& i\left(\frac{d u_{j}}{d t}+\alpha u_{j}\right)-(A u)_{j}+u_{j} v_{j}=f_{j}(t)+\varepsilon a_{j} u_{j} \frac{d w_{j}^{1}}{d t}, \\
& \frac{d v_{j}}{d t}=\psi_{j}-\sigma v_{j}, \\
& \frac{d \psi_{j}}{d t}+(\mu-\sigma) \psi_{j}+[1-\sigma(\mu-\sigma)+A] v_{j}-\left|u_{j}\right|^{2}=g_{j}(t)+\varepsilon b_{j} \frac{d w_{j}^{2}}{d t},
\end{aligned}
$$

with initial conditions

$$
u_{j}(0)=u_{j 0}, \quad v_{j}(0)=v_{j 0}, \quad \psi_{j}(0)=v_{1 j 0}+\sigma v_{j 0},
$$

where $u=\left(u_{j}\right)_{j \in \mathbb{Z}^{n}} \in \mathbb{L}^{2}, v=\left(v_{j}\right)_{j \in \mathbb{Z}^{n}} \in \ell^{2}, \psi=\left(\psi_{j}\right)_{j \in \mathbb{Z}^{n}} \in \ell^{2}$, and the linear operator $A$ is defined by

$$
\begin{aligned}
(A u)_{j}= & 2 n u_{\left(j_{1}, j_{2}, \ldots, j_{n}\right)}-u_{\left(j_{1}-1, j_{2}, \ldots, j_{n}\right)}-u_{\left(j_{1}, j_{2}-1, \ldots, j_{n}\right)}-\cdots-u_{\left(j_{1}, j_{2}, \ldots, j_{n}-1\right)} \\
& -u_{\left(j_{1}+1, j_{2}, \ldots, j_{n}\right)}-u_{\left(j_{1}, j_{2}+1, \ldots, j_{n}\right)}-\cdots-u_{\left(j_{1}, j_{2}, \ldots, j_{n}+1\right)} .
\end{aligned}
$$

In fact, the linear operator $A$ has the following decomposition:

$$
A=A_{1}+A_{2}+\cdots+A_{n}
$$


and there exist bounded linear operators $B_{k}: \mathbb{H} \rightarrow \mathbb{H}$ defined by

$$
\left(B_{k} u\right)_{j}=\sum_{l=-b_{0}}^{b_{0}} h_{k, l} u_{j_{k l}}, \quad \forall u=\left(u_{j}\right)_{j \in \mathbb{Z}^{n}} \in \mathbb{H}, k=1,2, \ldots, n,
$$

where $j_{k l}=\left(j_{1}, j_{2}, \ldots, j_{k-1}, j_{k}+l, j_{k+1}, \ldots, j_{n}\right) \in \mathbb{Z}^{n}$, such that

$$
A_{k}=B_{k}^{*} B_{k}=B_{k} B_{k}^{*}, \quad\left\|B_{k}\right\|_{0} \leq C \text { (constant), } \quad k=1,2, \ldots, n,
$$

where $\|\cdot\|_{0}$ denotes the norm of operator in the set of linear operators from $\mathbb{H}$ into itself and $B_{k}^{*}$ is the adjoint operator of $B_{k}, k=1,2, \ldots, n$, that is,

$$
\left(B_{k}^{*} u\right)_{j}=\sum_{l=-b_{0}}^{b_{0}} h_{k,-l} u_{j_{k l}}, \quad\left(B_{k} u, v\right)=\left(u, B_{k}^{*} v\right), \quad \forall u=\left(u_{j}\right)_{j \in \mathbb{Z}^{n}}, v \in \mathbb{H}, k=1,2, \ldots, n .
$$

Let $W^{1}(t)=\sum_{j \in \mathbb{Z}^{n}} a_{j} w_{j}^{1}(t) e^{j}$ and $W^{2}(t)=\sum_{j \in \mathbb{Z}^{n}} b_{j} w_{j}^{2}(t) e^{j}$, where $\left(a_{j}\right)_{j \in \mathbb{Z}^{n}},\left(b_{j}\right)_{j \in \mathbb{Z}^{n}} \in \ell^{2}$. Here $\left\{e^{j}\right\}$ denotes the standard complete orthonormal system in $\ell^{2}$, which means that the $j$ th component of $e^{j}$ is 1 and all other elements are 0 . Then $W^{1}(\cdot)$ and $W^{2}(\cdot)$ are $\ell^{2}$-valued $Q$-Wiener processes. It is obvious that $E W^{1}(t)=0$ and $E W^{2}(t)=0$. For details we refer to [32].

We abstract (2.1) as a stochastic ordinary differential equations with respect to time $t$ in $E=\mathbb{L}^{2} \times \ell^{2} \times \ell^{2}$. Let $a=\left(a_{j}\right)_{j \in \mathbb{Z}^{n}}, b=\left(b_{j}\right)_{j \in \mathbb{Z}^{n}}, v=\left(v_{j}\right)_{j \in \mathbb{Z}^{n}}, \psi=\left(\psi_{j}\right)_{j \in \mathbb{Z}^{n}}, f=\left(f_{j}\right)_{j \in \mathbb{Z}^{n}}$ and $g=\left(g_{j}\right)_{j \in \mathbb{Z}^{n}}$. Then the equations in (2.1) can be written as the following integral equations:

$$
\left\{\begin{array}{l}
u(t)=u_{0}+\int_{0}^{t}[i u(s) v(s)-\alpha u(s)-i A u(s)-i f(s)] d s-i \int_{0}^{t} u(s) d W^{1}, \\
v(t)=v_{0}+\int_{0}^{t}[\psi(s)-\sigma v(s)] d s \\
\psi(t)=\psi_{0}+\int_{0}^{t}\left[(\sigma-\mu) \psi(s)+(\sigma(\mu-\sigma)-A-1) v(s)+|u|^{2}+g(s)\right] d s+W^{2} .
\end{array}\right.
$$

For our purpose we introduce the probability space as

$$
\Omega=\left\{\omega \in \mathbb{C}\left(\mathbb{R}, \ell^{2}\right): \omega(0)=0\right\}
$$

endowed with the compact open topology [29]. $\mathbb{P}$ is the corresponding Wiener measure and $\mathbb{F}$ is the $\mathbb{P}$-completion of the Borel $\sigma$-algebra on $\Omega$.

Let $\theta_{t} \omega(\cdot)=\omega(\cdot+t)-\omega(t), t \in \mathbb{R}$. Then $\left(\Omega, \mathbb{F}, \mathbb{P},\left(\theta_{t}\right)_{t \in \mathbb{R}}\right)$ is a metric dynamical system with the filtration $\mathbb{F}_{t}:=\bigvee_{s \leq t} \mathbb{F}_{s}^{t}, t \in \mathbb{R}$, where $\mathbb{F}_{s}^{t}=\sigma\left\{W\left(t_{2}\right)-W\left(t_{1}\right): s \leq t_{1} \leq t_{2} \leq t\right\}$ is the smallest $\sigma$-algebra generated by the random variable $W\left(t_{2}\right)-W\left(t_{1}\right)$ for all $t_{1}, t_{2}$ such that $s \leq t_{1} \leq t_{2} \leq t$; see [29] for more details.

\section{The existence of a random attractor}

In this section, we study the dynamics of solutions of Klein-Gordon-Schrödinger lattice system under the $\varepsilon$-random perturbation (1.1). Then we apply Proposition 2.1 to prove the existence of a global random attractor for (1.1). In order to show the existence of a global solutions of system (2.2), we first change (2.2) into deterministic equations. Due to the special linear multiplicative noise, the first equation in system (2.1) can be reduced to an 
equation with random coefficients by a suitable change of variable. Consider the process $z(t)=e^{i \varepsilon W^{1}(t)}$, which satisfies the stochastic differential equation

$$
d z(t)=\frac{1}{2} z(t) d t+i \varepsilon z(t) d W^{1} .
$$

The process $\tilde{u}(t)=z(t) u(t)$ obeys the random differential equation

$$
i \frac{d \tilde{u}}{d t}+i\left(\alpha-\frac{1}{2}\right) \tilde{u}-(A \tilde{u})+\tilde{u} v-f(t) z(t)=0 .
$$

Lemma 3.1 Assume $f(t)=\left(f_{j}(t)\right)_{j \in \mathbb{Z}^{n}} \in \mathbb{C}_{b}\left(\mathbb{R}, \ell^{2}\right)$. Then the solution of the first equation in (3.1) satisfies

$$
\|\tilde{u}\|^{2} \leq e^{-\alpha t}\left\|\tilde{u}_{0}\right\|^{2}+\frac{1}{\alpha}\|f\|^{2}, \quad t>0, \text { a.a. } \omega \in \Omega,
$$

with $\|f\|=\sup _{t \in \mathbb{R}}|f(t)|^{2}$.

We denote $\tilde{\psi}(t)=\psi(t)-\varepsilon W^{2}(t)$, then system (2.2) can be changed into the following system:

$$
\left\{\begin{aligned}
\tilde{u}(t)= & \tilde{u}_{0}+\int_{0}^{t}\left[i \tilde{u}(s) v(s)-i\left(\alpha-\frac{1}{2}\right) \tilde{u}(s)-i A \tilde{u}(s)-i f(s) z(s)\right] d s \\
v(t)= & v_{0}+\int_{0}^{t}\left[\tilde{\psi}(s)-\sigma v(s)+\varepsilon W^{2}(s)\right] d s, \\
\tilde{\psi}(t)= & \psi_{0}+\int_{0}^{t}\left[(\sigma-\mu) \tilde{\psi}(s)+(\sigma(\mu-\sigma)-A-1) v(s)+|u|^{2}+g(s)\right. \\
& \left.+\varepsilon(\sigma-\mu) W^{2}(s)\right] d s .
\end{aligned}\right.
$$

For each fixed $\omega \in \Omega$, system (3.3) is a deterministic equation, and we have the following result.

Theorem 3.1 For any $T>0$, system (2.2) is well-posed and admits a unique solution $(u(t), v(t), \psi(t)) \in \mathbb{L}^{2}(\Omega ; \mathbb{C}([0, T] ; E))$. Moreover, the solution of $(2.2)$ depends continuously on the initial data $\left(u_{0}, v_{0}, \psi_{0}\right)$.

Proof By the standard existence theorem for ODEs, it follows that system (3.3) possesses a local solution $(\tilde{u}(t), v(t), \tilde{\psi}(t)) \in \mathbb{C}(0, T ; E)$, where $\left[0, T_{\max }\right)$ is the maximal interval of existence of the solution of (3.3). Now, we prove that this local solution is a global solution. Let $\omega \in \Omega$, from (3.3) it follows that

$$
\begin{aligned}
\|\tilde{u}(t)\|^{2}+\|v(t)\|^{2}+\|\tilde{\psi}(t)\|^{2} \\
=\left\|\tilde{u}_{0}\right\|^{2}+\left\|v_{0}\right\|^{2}+\left\|\psi_{0}\right\|^{2} \\
-(2 \alpha-1) \int_{0}^{t}(\tilde{u}(s), \tilde{u}(s)) d s-2 \int_{0}^{t} \operatorname{Im}(f(s) z(s), \tilde{u}(s)) d s+2 \int_{0}^{t}(\tilde{\psi}(s), v(s)) d s \\
+2 \int_{0}^{t}\left(\varepsilon W^{2}(s), v(s)\right) d s-2 \int_{0}^{t}(\sigma v(s), v(s)) d s+2 \int_{0}^{t}((\sigma-\mu) \tilde{\psi}(s), \tilde{\psi}(s)) d s \\
+2 \int_{0}^{t}(g(s), \tilde{\psi}(s)) d s+2 \int_{0}^{t}((\sigma(\mu-\sigma)-A-1) v(s), \tilde{\psi}(s)) d s \\
+2 \int_{0}^{t}\left(|u|^{2}, \tilde{\psi}(s)\right) d s+2 \int_{0}^{t}\left(\varepsilon(\sigma-\mu) W^{2}(s), \tilde{\psi}(s)\right) d s \quad \text { (taking the real part). }
\end{aligned}
$$


By the definition of the linear operator $A$, we have

$$
\begin{aligned}
-(A v, \tilde{\psi}) & =-\sum_{k=1}^{n}\left(A_{k} v, \tilde{\psi}\right)=-\sum_{k=1}^{n}\left(B_{k}^{*} B_{k} v, \tilde{\psi}\right)=\sum_{k=1}^{n}\left(B_{k} v, B_{k} \tilde{\psi}\right) \\
& \leq \frac{1}{2} \sum_{k=1}^{n}\left(\left\|B_{k} v\right\|^{2}+\left\|B_{k} \tilde{\psi}\right\|^{2}\right) \leq \frac{n C^{2}}{2}\left(\|v\|^{2}+\|\tilde{\psi}\|^{2}\right) .
\end{aligned}
$$

By the Young inequality, direct computation shows that

$$
\begin{aligned}
& -2 \operatorname{Im}(f z, \tilde{u}) \leq \frac{2}{\alpha}\|f\|^{2}\|z\|^{2}+\frac{\alpha}{2}\|\tilde{u}\|^{2} \leq \frac{1}{\alpha}\left(\|f\|^{4}+\left\|W^{1}\right\|^{4}\right)+\frac{\alpha}{2}\|\tilde{u}\|^{2}, \\
& 2 \varepsilon\left(W^{2}, v\right) \leq \frac{4 \varepsilon^{2}}{\sigma}\left\|W^{2}\right\|^{2}+\frac{\sigma}{4}\|v\|^{2} \\
& 2(g, \tilde{\psi}) \leq \frac{4}{\mu-\sigma}\|g\|^{2}+\frac{\mu-\sigma}{4}\|\tilde{\psi}\|^{2} \\
& 2((\sigma(\mu-\sigma)) v, \tilde{\psi}) \leq 4\left(\sigma^{2}(\mu-\sigma)\right)\|v\|^{2}+\frac{\mu-\sigma}{4}\|\tilde{\psi}\|^{2}, \\
& 2\left(|u|^{2}, \tilde{\psi}\right) \leq \frac{4}{\mu-\sigma}\|u\|^{4}+\frac{\mu-\sigma}{4}\|\tilde{\psi}\|^{2}, \\
& 2 \varepsilon\left((\sigma-\mu) W^{2}, \tilde{\psi}\right) \leq 4 \varepsilon^{2}(\mu-\sigma)\left\|W^{2}\right\|^{2}+\frac{\mu-\sigma}{4}\|\tilde{\psi}\|^{2} .
\end{aligned}
$$

Combining the above inequalities with Lemma 3.1, we obtain

$$
\begin{aligned}
& \|\tilde{u}(t)\|^{2}+\|v(t)\|^{2}+\|\tilde{\psi}(t)\|^{2} \\
& \leq\left\|\tilde{u}_{0}\right\|^{2}+\left\|v_{0}\right\|^{2}+\left\|\tilde{\psi}_{0}\right\|^{2} \\
& \quad-C_{0} \int_{0}^{t}\left(\|\tilde{u}(s)\|^{2}+\|v(s)\|^{2}+\|\tilde{\psi}(s)\|^{2}\right) d s \\
& \quad+C_{1} \int_{0}^{t}\left(\|f\|^{2}+\|g\|^{2}+\left\|W^{1}\right\|^{4}+\left\|W^{2}\right\|^{2}\right) d s \\
& \leq\left\|\tilde{u}_{0}\right\|^{2}+\left\|v_{0}\right\|^{2}+\left\|\tilde{\psi}_{0}\right\|^{2}+C_{1} \int_{0}^{t}\left(\left\|W^{1}\right\|^{4}+\left\|W^{2}\right\|^{2}\right) d s \\
& \quad+C_{1} \int_{0}^{t}\|f\|^{2}+\|g\|^{2} d s,
\end{aligned}
$$

where $C_{0}, C_{1}$ are constants depending on $\alpha, \sigma, \mu, \varepsilon$, and $\|f\|^{2}$. By the Gaussian property of $W^{1}$ and $W^{2},(3.4)$ implied that system (3.3) admits the global solution $(\tilde{u}(t), v(t), \tilde{\psi}(t)) \in$ $\mathbb{L}^{2}(\Omega ; \mathbb{C}([0, T] ; E))$. The proof is completed.

From the definition $\left(\theta_{t}\right)_{t \in \mathbb{R}}$, we know

$$
W(t+h, \omega)=W\left(t, \theta_{h} \omega\right)+W(h, \omega), \quad \forall t, h \in \mathbb{R}
$$

and combining the above theorem we have the following result.

Theorem 3.2 System (2.2) generates a continuous random dynamical system ( $\phi(t$, $\left.\left.\theta_{-t} \omega\right)\right)_{t \geq 0}$ over $\left(\Omega, \mathbb{F}, \mathbb{P},\left(\theta_{t}\right)_{t \in \mathbb{R}}\right)$. 
The proof is similar to that of Theorem 3.2 in [24], so we omit it.

Now, we prove the existence of a random attractor for system (2.2). By Proposition 2.1, we first prove that RDS $\phi$ possesses a bounded absorbing set $\mathbb{K}(\omega)$. We introduce an Ornstein-Uhlenbeck process in $\ell^{2}$ on the metric dynamical system $\left(\Omega, \mathbb{F}, \mathbb{P}, \theta_{t}\right)$ given by a Wiener process:

$$
\begin{aligned}
& z^{1}\left(\theta_{t} \omega\right)=-v \int_{-\infty}^{0} e^{v h} \theta_{t} \omega(h) d h, \quad t \in \mathbb{R}, \\
& z^{2}\left(\theta_{t} \omega\right)=-\lambda \int_{-\infty}^{0} e^{\lambda h} \theta_{t} \omega(h) d h, \quad t \in \mathbb{R},
\end{aligned}
$$

where $v$ and $\lambda$ are positive. The above integral exists in the sense that for any path $\omega$ with a subexponential growth $z^{1}, z^{2}$ solve the following Itô equations:

$$
\begin{aligned}
& d z^{1}+v z^{1} d t=d W^{1}(t) \\
& d z^{2}+\lambda z^{2} d t=d W^{2}(t) .
\end{aligned}
$$

In fact, the mapping $t \rightarrow z^{i}\left(\theta_{t} \omega\right), i=1,2$, are the Ornstein-Uhlenbeck process. Furthermore, there exists a $\theta_{t}$ invariant set $\Omega^{\prime} \subset \Omega$ of full $\mathbb{P}$ measure such that:

(1) the mappings $t \rightarrow z^{i}\left(\theta_{t} \omega\right), i=1,2$, are continuous for each $\omega \in \Omega^{\prime}$;

(2) the random variables $\left\|z^{i}(\omega)\right\|, i=1,2$, are tempered.

Lemma 3.2 There exists a $\theta_{t}$ invariant set $\Omega^{\prime} \subset \Omega$ of full $\mathbb{P}$ measure and an absorbing random set $\mathbb{K}(\omega), \omega \in \Omega^{\prime}$, for the random dynamical system $\left(\phi\left(t, \theta_{-t} \omega\right)\right)_{t \geq 0}$.

Proof We use the estimates in Theorem 3.1. By (3.4), we have

$$
\begin{aligned}
\frac{d\left(\|\tilde{u}(t)\|^{2}+\|v(t)\|^{2}+\|\tilde{\psi}(t)\|^{2}\right)}{d t} \leq & -C_{0}\left(\|\tilde{u}(t)\|^{2}+\|v(t)\|^{2}+\|\tilde{\psi}(t)\|^{2}\right) \\
& +C_{1} \rho\left(\theta_{t} \omega\right)
\end{aligned}
$$

where $\rho\left(\theta_{t} \omega\right)=\|f\|^{2}+\|g\|^{2}+\left\|W^{1}(t)\right\|^{4}+\left\|W^{2}(t)\right\|^{2}$.

By Gronwall's lemma, it follows that

$$
\begin{aligned}
\|\tilde{u}(t)\|^{2}+\|v(t)\|^{2}+\|\tilde{\psi}(t)\|^{2} \leq & \left(\left\|\tilde{u}_{0}\right\|^{2}+\left\|v_{0}\right\|^{2}+\left\|\psi_{0}\right\|^{2}\right) e^{-C_{0} t} \\
& +\int_{0}^{t} e^{-C_{0}(t-s)} \rho\left(\theta_{s} \omega\right) d s
\end{aligned}
$$

Replace $\omega$ by $\theta_{-t} \omega$ in the above inequality to construct the radius of the absorbing set and define

$$
\varrho^{2}(\omega)=4 \lim _{t \rightarrow \infty} \int_{0}^{t} e^{-C_{0}(t-s)} \rho\left(\theta_{s-t} \omega\right) d s=4 \lim _{t \rightarrow \infty} \int_{-t}^{0} e^{-C_{0} s} \rho\left(\theta_{s} \omega\right) d s
$$

Define

$$
R(\omega)=\varrho^{2}(\omega)+\frac{4}{\alpha}\|f\|^{2}\left\|W^{1}\right\|^{4}+\left\|W^{2}\right\|^{2} .
$$


Then $\mathbb{K}(\omega) \triangleq \mathbb{K}(0, R(\omega))$ is a tempered ball by the property of $W^{1}, W^{2}$, and, for any $B \in \mathbb{D}$, $\omega \in \Omega$. Here, $\mathbb{D}$ denotes the collection of all tempered random set of Hilbert space $H$. This completes the proof.

Lemma 3.3 Let $\left(u_{0}, v_{0}, \psi_{0}\right) \in \mathbb{K}(\omega)$, the absorbing set given in Lemma 3.2. Then for every $\varepsilon>0$ and $\mathbb{P}$-a.e. $\omega \in \Omega$, there exist $T(\varepsilon, \omega)>0$ and $N(\varepsilon, \omega)>0$ such that the solution $(u, v, \psi)$ of system (2.2) satisfies

$$
\sum_{\|j\|>N(\varepsilon, \omega)}\left[\left|u_{n}\left(t, \theta_{-t} \omega\right)\right|^{2}+\left|v_{n}\left(t, \theta_{-t} \omega\right)\right|^{2}+\left|\psi_{n}\left(t, \theta_{-t} \omega\right)\right|^{2}\right] \leq \varepsilon, \quad \forall t \geq T(\varepsilon, \omega)
$$

where $\|j\|=\max _{1 \leq k \leq n}\left|j_{k}\right|$ for $j=\left(j_{1}, j_{2}, \ldots, j_{n}\right) \in \mathbb{Z}^{n}$.

Proof Let $\eta(x) \in \mathbb{C}\left(\mathbb{R}_{+},[0,1]\right)$ be a cut-off function satisfying

$$
\eta(x)=0, \quad \text { for all } x \in[0,1] ; \quad \eta(x)=1, \quad \text { for all } x \in[2,+\infty),
$$

and $\left|\eta^{\prime}(x)\right| \leq \eta_{0}$ (a positive constant).

Let $M$ be a suitable large integer. Taking the inner product of (3.3) with $\left(\eta\left(\frac{\|j\|}{M}\right) \tilde{u}_{j}\right)_{j \in \mathbb{Z}^{n}}$, $\left(\eta\left(\frac{\|j\|}{M}\right) v_{j}\right)_{j \in \mathbb{Z}^{n}}$, and $\left(\eta\left(\frac{\|j\|}{M}\right) \tilde{\psi}_{j}\right)_{j \in \mathbb{Z}^{n}}$, we get

$$
\begin{aligned}
& \frac{d}{d t} \sum_{j \in \mathbb{Z}^{n}} \eta\left(\frac{\|j\|}{M}\right)\left|\tilde{u}_{j}\right|^{2} \\
& \quad=-2 \alpha \sum_{j \in \mathbb{Z}^{n}} \eta\left(\frac{\|j\|}{M}\right)\left|\tilde{u}_{j}\right|^{2}-2 \operatorname{Im} \sum_{j \in \mathbb{Z}^{n}} \eta\left(\frac{\|j\|}{M}\right)\left(f_{j} z_{j}, \tilde{u}_{j}\right) \quad \text { (taking the real part), } \\
& \frac{d}{d t} \sum_{j \in \mathbb{Z}^{n}} \eta\left(\frac{\|j\|}{M}\right)\left|v_{j}\right|^{2} \\
& \quad=2 \sum_{j \in \mathbb{Z}^{n}} \eta\left(\frac{\|j\|}{M}\right)\left(\tilde{\psi}_{j}, v_{j}\right)-2 \sigma \sum_{j \in \mathbb{Z}^{n}} \eta\left(\frac{\|j\|}{M}\right)\left|v_{j}\right|^{2}+2 \varepsilon \sum_{j \in \mathbb{Z}^{n}} \eta\left(\frac{\|j\|}{M}\right)\left(W_{j}^{2}, v_{j}\right)
\end{aligned}
$$

and

$$
\begin{aligned}
& \frac{d}{d t} \sum_{j \in \mathbb{Z}^{n}} \eta\left(\frac{\|j\|}{M}\right)\left|\tilde{\psi}_{j}\right|^{2} \\
& =2(\sigma-\mu) \sum_{j \in \mathbb{Z}^{n}} \eta\left(\frac{\|j\|}{M}\right)\left|\tilde{\psi}_{j}\right|^{2}-2 \sum_{j \in \mathbb{Z}^{n}} \eta\left(\frac{|j|}{M}\right)\left(A v_{j}, \tilde{\psi}_{j}\right) \\
& \quad+2[\sigma(\mu-\sigma)-1] \sum_{j \in \mathbb{Z}^{n}} \eta\left(\frac{\|j\|}{M}\right)\left(v_{j}, \tilde{\psi}_{j}\right) \\
& \quad+2 \sum_{j \in \mathbb{Z}^{n}} \eta\left(\frac{\|j\|}{M}\right)\left(\left|u_{j}\right|^{2}, \tilde{\psi}_{j}\right)+2 \sum_{j \in \mathbb{Z}^{n}} \eta\left(\frac{\|j\|}{M}\right)\left(g_{j}, \tilde{\psi}_{j}\right) \\
& \quad+2 \varepsilon(\sigma-\mu) \sum_{j \in \mathbb{Z}^{n}} \eta\left(\frac{\|j\|}{M}\right)\left(W_{j}^{2}, \tilde{\psi}_{j}\right) .
\end{aligned}
$$


We also use the estimates in Theorem 3.1. Similar to (3.4), it follows that, for fixed constant $T_{k}>0$,

$$
\begin{aligned}
& \sum_{j \in \mathbb{Z}^{n}} \eta\left(\frac{\|j\|}{M}\right)\left(\left|\tilde{u}_{j}\right|^{2}+\left|v_{j}\right|^{2}+\left|\tilde{\psi}_{j}\right|^{2}\right) \\
& \leq e^{-C_{0}\left(t-T_{K}\right)} \sum_{j \in \mathbb{Z}^{n}} \eta\left(\frac{\|j\|}{M}\right)\left|\tilde{u}_{j}\left(T_{k}, \omega\right)\right|^{2} \\
& \quad+e^{-C_{0}\left(t-T_{K}\right)} \sum_{j \in \mathbb{Z}^{n}} \eta\left(\frac{\|j\|}{M}\right)\left(\left|v_{j}\left(T_{k}, \omega\right)\right|^{2}+\left|\tilde{\psi}_{j}\left(T_{k}, \omega\right)\right|^{2}\right) \\
& \quad+C_{1} \int_{T_{k}}^{t} e^{C_{0}(s-t)} \sum_{\|j\| \geq M}\left(\left|z_{j}^{1}\left(\theta_{s} \omega\right)\right|^{4}+\left|z_{j}^{2}\left(\theta_{s} \omega\right)\right|^{2}\right) d s \\
& \quad+C_{1} \int_{T_{k}}^{t} e^{C_{0}(s-t)} \sum_{\|j j\| \geq M}\left(\left|f_{j}\right|^{2}+\left|g_{j}\right|^{2}\right) d s .
\end{aligned}
$$

Replace $\omega$ by $\theta_{-t} \omega$ in (3.5). Then we estimate each of the terms on the right-hand of (3.5), and it follows that

$$
\begin{aligned}
& e^{-C_{0}\left(t-T_{K}\right)} \sum_{j \in \mathbb{Z}^{n}} \eta\left(\frac{\|j\|}{M}\right)\left(\left|\tilde{u}_{j}\left(T_{k}, \theta_{-t} \omega\right)\right|^{2}+\left|v_{j}\left(T_{k}, \theta_{-t} \omega\right)\right|^{2}+\left|\tilde{\psi}_{j}\left(T_{k}, \theta_{-t} \omega\right)\right|^{2}\right) \\
& \leq e^{-C_{0}\left(t-T_{K}\right)}\left[\left(\left|u_{0}\left(\theta_{-t} \omega\right)\right|^{2}+\left|v_{0}\left(\theta_{-t} \omega\right)\right|^{2}+\left|\psi_{0}\left(\theta_{-t} \omega\right)\right|^{2}\right) e^{-C_{0} T_{k}}\right. \\
& \quad+\int_{0}^{T_{K}} e^{-C_{0}\left(T_{K}-s\right)}\left(\left|z^{1}\left(\theta_{s-t} \omega\right)\right|^{4}+\left|z^{2}\left(\theta_{s-t} \omega\right)\right|^{2}\right) d s \\
& \left.\quad+e^{-C_{0}\left(t-T_{K}\right)} \int_{0}^{T_{K}} e^{-C_{0}\left(T_{K}-s\right)}\left(|f|^{2}+|g|^{2}\right) d s\right] .
\end{aligned}
$$

Since $\left\|z^{i}(\omega)\right\|, i=1,2$, are tempered and $z^{i}\left(\theta_{t} \omega\right), i=1,2$, are continuous in $t$, there is a tempered function $r(\omega)>0$, such that

$$
\left\|z^{1}\left(\theta_{t} \omega\right)\right\|^{4}+\left\|z^{2}\left(\theta_{t} \omega\right)\right\|^{2} \leq r\left(\theta_{t} \omega\right) .
$$

Combining (3.6) with (3.7), there is a constant $T_{1}(\varepsilon, \omega)>T_{K}$, such that

$$
\begin{aligned}
& e^{-C_{0}\left(t-T_{K}\right)} \sum_{j \in \mathbb{Z}^{n}} \eta\left(\frac{\|j\|}{M}\right)\left(\left|\tilde{u}_{j}\left(T_{k}, \theta_{-t} \omega\right)\right|^{2}+\left|v_{j}\left(T_{k}, \theta_{-t} \omega\right)\right|^{2}+\left|\tilde{\psi}_{j}\left(T_{k}, \theta_{-t} \omega\right)\right|^{2}\right) \\
& \quad \leq \frac{\varepsilon}{3} .
\end{aligned}
$$

Next, we estimate

$$
C_{1} \int_{T_{k}}^{t} e^{C_{0}(s-t)} \sum_{\|j\| \geq M}\left(\left|z_{j}^{1}\left(\theta_{s-t} \omega\right)\right|^{4}+\left|z_{j}^{2}\left(\theta_{s-t} \omega\right)\right|^{2}\right) d s .
$$


Let $T * \geq \frac{1}{C_{0}} \ln \left(\frac{6 C_{1} r(\omega)}{C_{0} \varepsilon}\right)$ and $N_{1}(\varepsilon, \omega)$ be fixed positive constants. Then, for $t>T *+T_{k}$ and $M>N_{1}(\varepsilon, \omega)$, we have

$$
\begin{aligned}
& C_{1} \int_{T_{k}}^{t} e^{C_{0}(s-t)} \sum_{\|j\| \geq M}\left(\left|z_{j}^{1}\left(\theta_{s-t} \omega\right)\right|^{4}+\left|z_{j}^{2}\left(\theta_{s-t} \omega\right)\right|^{2}\right) d s \\
& =C_{1} \int_{-T *}^{0} e^{C_{0} \xi} \sum_{\|j\| \geq M}\left(\left|z_{j}^{1}\left(\theta_{\xi} \omega\right)\right|^{4}+\left|z_{j}^{2}\left(\theta_{\xi} \omega\right)\right|^{2}\right) d \xi \\
& \quad+C_{1} \int_{T_{K}-t}^{-T *} e^{C_{0} \xi}\left(\left|z_{j}^{1}\left(\theta_{\xi} \omega\right)\right|^{4}+\left|z_{j}^{2}\left(\theta_{\xi} \omega\right)\right|^{2}\right) d \xi \\
& \leq C_{1} \int_{-T *}^{0} e^{C_{0} \xi} \sum_{\|j\| \geq M}\left(\left|z_{j}^{1}\left(\theta_{\xi} \omega\right)\right|^{4}+\left|z_{j}^{2}\left(\theta_{\xi} \omega\right)\right|^{2}\right) d \xi+\frac{C_{1}}{C_{0}} r(\omega) e^{-C_{0} T *} \\
& \leq \frac{\varepsilon}{6}+\frac{\varepsilon}{6}=\frac{\varepsilon}{3} \quad \text { (by the Lebesgue theorem). }
\end{aligned}
$$

Since $f(t) \in \mathbb{C}_{b}\left(\mathbb{R}, \ell^{2}\right)$ and $g(t) \in \mathbb{C}_{b}\left(\mathbb{R}, \ell^{2}\right)$, there exists $N_{2}(\varepsilon, \omega)$ such that for $M>$ $N_{2}(\varepsilon, \omega)$

$$
C_{1} \int_{T_{k}}^{t} e^{C_{0}(s-t)} \sum_{\|j\| \geq M}\left(\left|f_{j}\right|^{2}+\left|g_{j}\right|^{2}\right) d s \leq \frac{\varepsilon}{3} .
$$

Therefore, let

$$
\tilde{T}(\varepsilon, \omega)=\max \left\{T_{1}(\varepsilon, \omega), T *(\varepsilon, \omega)\right\} \quad \text { and } \quad \tilde{N}(\varepsilon, \omega)=\max \left\{N_{1}(\varepsilon, \omega), N_{2}(\varepsilon, \omega)\right\} .
$$

Then, for $t>\tilde{T}(\varepsilon, \omega)$ and $M>\tilde{N}(\varepsilon, \omega)$, we obtain

$$
\sum_{\|j\|>M}\left(\left|\tilde{u}_{j}\left(t, \theta_{t} \omega\right)\right|^{2}+\left|v_{j}\left(t, \theta_{t} \omega\right)\right|^{2}+\left|\tilde{\psi}_{j}\left(t, \theta_{t} \omega\right)\right|^{2}\right) \leq \varepsilon
$$

Direct computation shows that

$$
\|u\|^{2}+\|v\|^{2}+\|\psi\|^{2} \leq 2\left(\|\tilde{u}\|^{2}+\|v\|^{2}+\|\tilde{\psi}\|^{2}\right)+4\left\|z^{2}\left(\theta_{t} \omega\right)\right\|^{2} .
$$

Therefore, we obtain

$$
\sum_{\|j\|>M}\left(\left|u_{j}\left(t, \theta_{t} \omega\right)\right|^{2}+\left|v_{j}\left(t, \theta_{t} \omega\right)\right|^{2}+\left|\psi_{j}\left(t, \theta_{t} \omega\right)\right|^{2}\right) \leq \varepsilon .
$$

This completes the proof.

Lemma 3.4 The random dynamical system $\left(\phi\left(t, \theta_{-t} \omega\right)\right)_{t \geq 0}$ is asymptotically compact.

Proof We use the method of [18]. Let $\omega \in \Omega$. Consider a sequence $\left(t_{n}\right)_{n \in \mathbb{N}}$ with $t_{n} \rightarrow \infty$ as $n \rightarrow \infty$. Since $\mathbb{K}(\omega)$ is a bounded absorbing set, for large $n,\left(u_{n}, v_{n}, \psi_{n}\right)=\phi\left(t_{n}, \theta_{-t_{n}} \omega\right)\left(u_{0}, v_{0}\right.$, $\left.\psi_{0}\right) \in \mathbb{K}(\omega)$, where $\left(u_{0}, v_{0}, \psi_{0}\right) \in \mathbb{K}(\omega)$. Then there exist $(u, v, \psi) \in E$ and a sequence, denoted by $\left(u_{n}, v_{n}, \psi_{n}\right)$, such that

$$
\left(u_{n}, v_{n}, \psi_{n}\right) \rightarrow(u, v, \psi) \quad \text { weak in } E .
$$


Next, we show that the above weak convergence is actually strong convergence in $E$.

From Lemma 3.3, for any $\varepsilon>0$, there exist positive constants $N_{3}(\varepsilon, \omega)$ and $\tilde{M}_{1}$ such that for $n>\tilde{M}_{1}$,

$$
\sum_{\|j\|>N_{3}}\left(\left|u_{i n}\left(t_{n}, \theta_{t_{n}} \omega\right)\right|^{2}+\left|v_{j n}\left(t_{n}, \theta_{t_{n}} \omega\right)\right|^{2}+\left|\psi_{j n}\left(t_{n}, \theta_{t_{n}} \omega\right)\right|^{2}\right) \leq \frac{\varepsilon}{6}
$$

Since $(u, v, \psi) \in E$, there exists $N_{4}(\varepsilon, \omega)>0$ such that

$$
\sum_{\|j\| \geq N_{4}}\left(\left|u_{j}\right|^{2}+\left|v_{j}\right|^{2}+\left|\psi_{j}\right|^{2}\right) \leq \frac{\varepsilon}{6}
$$

Let $\tilde{N}(\varepsilon, \omega)=\max \left\{N_{3}(\varepsilon, \omega), N_{4}(\varepsilon, \omega)\right\}$, then, from (3.11), there exists $\tilde{M}_{2}>0$ such that for $n>\tilde{M}_{2}$,

$$
\sum_{\|j\| \leq \tilde{N}}\left(\left|u_{j n}-u\right|^{2}+\left|v_{j n}-v\right|^{2}+\left|\psi_{j n}-\psi\right|^{2}\right) \leq \frac{\varepsilon}{3} .
$$

By (3.12)-(3.14), we find that, for $n>\tilde{M}=\max \left\{\tilde{M}_{1}, \tilde{M}_{2}\right\}$,

$$
\begin{aligned}
& \left\|u_{j}\left(t_{n}, \theta_{-t_{n}} \omega\right)-u\right\|^{2}+\left\|v_{j}\left(t_{n}, \theta_{-t_{n}}\right)-v\right\|^{2}+\left\|\psi_{j}\left(t_{n}, \theta_{-t_{n}}\right)-\psi\right\|^{2} \\
& =\sum_{\|j\| \leq \tilde{N}}\left(\left|u_{j n}\left(t_{n}, \theta_{-t_{n}} \omega\right)-u_{j}\right|^{2}+\left|v_{j n}\left(t_{n}, \theta_{-t_{n}} \omega\right)-v_{j}\right|^{2}+\left|\psi_{j n}\left(t_{n}, \theta_{-t_{n}} \omega\right)-\psi_{j}\right|^{2}\right) \\
& \quad+\sum_{\|j j\| \tilde{N}}\left(\left|u_{j n}\left(t_{n}, \theta_{-t_{n}} \omega\right)-u_{j}\right|^{2}+\left|v_{j n}\left(t_{n}, \theta_{-t_{n}} \omega\right)-v_{j}\right|^{2}+\left|\psi_{j n}\left(t_{n}, \theta_{-t_{n}} \omega\right)-\psi_{j}\right|^{2}\right) \\
& \leq \sum_{\|j\| \leq \tilde{N}}\left(\left|u_{j n}\left(t_{n}, \theta_{-t_{n}} \omega\right)-u_{j}\right|^{2}+\left|v_{j n}\left(t_{n}, \theta_{-t_{n}} \omega\right)-v_{j}\right|^{2}+\left|\psi_{j n}\left(t_{n}, \theta_{-t_{n}} \omega\right)-\psi_{j}\right|^{2}\right) \\
& \quad+2 \sum_{\| j j>\tilde{N}}\left(\left|u_{j n}\left(t_{n}, \theta_{-t_{n}} \omega\right)\right|^{2}+\left|v_{j n}\left(t_{n}, \theta_{-t_{n}} \omega\right)\right|^{2}+\left|\psi_{j n}\left(t_{n}, \theta_{-t_{n}} \omega\right)\right|^{2}\right) \\
& \quad+2 \sum_{\|j\| \tilde{N}}\left(\left|u_{j}\right|^{2}+\left|v_{j}\right|^{2}+\left|\psi_{j}\right|^{2}\right) \\
& \leq \frac{2 \varepsilon}{3}+\frac{2 \varepsilon}{6}+\frac{2 \varepsilon}{6} \leq \varepsilon .
\end{aligned}
$$

This completes the proof.

Now, combining Lemma 3.2, Lemma 3.4, and Proposition 2.1, we can easily obtain the following result.

Theorem 3.3 The random dynamical system $\left(\phi\left(t, \theta_{-t} \omega\right)\right)_{t \geq 0}$ possesses a global random attractor $A_{\varepsilon}$ in $E$.

\section{Upper semicontinuity of attractors}

This section studies the upper semicontinuity of random attractors for the stochastic Klein-Gordon-Schrödinger lattice system. The existence of global attractors for the KleinGordon-Schrödinger lattice system has been obtained by [6] for one dimension, and [16] 
for the high dimensional case. We assume $A_{0}$ is an attractor corresponding to the KleinGordon-Schrödinger lattice system, i.e., the case $\varepsilon=0$ of system (1.1). By Theorem 2 of [33], we only need to prove the following lemma for upper semicontinuity of attractors. In what follows, we take the vector form for brevity.

Lemma 4.1 Assume that $\left(u_{\varepsilon}\left(t, \omega, u_{0}\right), v_{\varepsilon}\left(t, \omega, v_{0}\right)\right)$ and $\left(u\left(t, u_{0}\right), v\left(t, v_{0}\right)\right)$ are the solutions of the perturbed lattice system (1.1) and the unperturbed lattice system (the case of $\varepsilon=0 \mathrm{in}$ (1.1)), respectively. Then, for $\mathbb{P}$-almost every $\omega \in \Omega$ and the absorbing set $\mathbb{B}$, we have

$$
\lim _{\varepsilon \rightarrow 0}\left|u_{\varepsilon}\left(t, \omega, u_{0}\right)-u\left(t, u_{0}\right)\right|=0, \quad \lim _{\varepsilon \rightarrow 0}\left|v_{\varepsilon}\left(t, \omega, v_{0}\right)-v\left(t, v_{0}\right)\right|=0, \quad t>0, \forall\left(u_{0}, v_{0}\right) \in \mathbb{B} .
$$

Proof Let $\gamma(t)=u_{\varepsilon}(t)-u(t), \delta(t)=v_{\varepsilon}(t)-v(t)$. Then $(\gamma(t), \delta(t))$ satisfies

$$
\begin{aligned}
& i\left(\frac{d \gamma}{d t}+\alpha \gamma\right)-A \gamma+u_{\varepsilon} v_{\varepsilon}-u v=\varepsilon u_{\varepsilon} \frac{d W^{1}}{d t}, \\
& \frac{d^{2} \delta}{d t^{2}}+A \delta+\mu \frac{d \delta}{d t}+\delta+|u|^{2}-\left|u_{\varepsilon}\right|^{2}=\varepsilon \frac{d W^{2}}{d t} .
\end{aligned}
$$

Now, we use the change of variables

$$
\rho(t)=\frac{d \delta}{d t}+\sigma \delta, \quad \tilde{\gamma}(t)=z(t) \gamma(t), \quad \tilde{\rho}=\rho(t)-\varepsilon W^{2}(t),
$$

where $\sigma \leq \min \{\mu, 1\}$ is a positive constant, and $z(t)=e^{i \varepsilon W^{1}}$.

Then we change (4.1) into

$$
\begin{aligned}
& i \frac{d \tilde{\gamma}}{d t}+i\left(\alpha-\frac{1}{2}\right) \tilde{\gamma}-A \tilde{\gamma}+u_{\varepsilon} \delta z+\tilde{\gamma} z=0, \\
& \frac{d \delta}{d t}+\sigma \delta=\tilde{\rho}+\varepsilon W^{2}, \\
& \frac{d \tilde{\rho}}{d t}+(\mu-\sigma) \tilde{\rho}+[A-\sigma(\mu-\sigma)+1] \delta+|u|^{2}-\left|u_{\varepsilon}\right|^{2}+\varepsilon(\sigma-\mu) W^{2}=0 .
\end{aligned}
$$

Taking the imaginary part of the inner product of the first equation in (4.2) with $\tilde{\gamma}$, we have

$$
\frac{d\|\tilde{\gamma}\|^{2}}{d t}+(2 \alpha-1)\|\tilde{\gamma}\|^{2}+2 \operatorname{Im}\left(u_{\varepsilon} \delta z, \tilde{\gamma}\right)+2 \operatorname{Im}(\tilde{\gamma} z, \tilde{\gamma})=0
$$

Taking the inner product of the second and third equation in (4.2) with $\delta$ and $\tilde{\rho}$, we have

$$
\frac{d\|\delta\|^{2}}{d t}+2 \sigma\|\delta\|^{2}=2(\tilde{\rho}, \delta)+2\left(\varepsilon W^{2}, \delta\right)
$$

and

$$
\begin{aligned}
& \frac{d\|\tilde{\rho}\|^{2}}{d t}+2(\mu-\sigma)\|\tilde{\rho}\|^{2}+2[A-\sigma(\mu-\sigma)+1](\delta, \tilde{\rho})+2\left(\left|u_{\varepsilon}\right|^{2}-|u|^{2}, \tilde{\rho}\right) \\
& \quad+2 \varepsilon(\sigma-\mu)\left(W^{2}, \tilde{\rho}\right)=0 .
\end{aligned}
$$


Summing up (4.3)-(4.5), we get

$$
\begin{aligned}
\frac{d}{d t}\left(\|\tilde{\gamma}\|^{2}+\|\delta\|^{2}+\|\tilde{\rho}\|^{2}\right)+(2 \alpha-1)\|\tilde{\gamma}\|^{2}+2 \sigma\|\delta\|^{2}+2(\mu-\sigma)\|\tilde{\rho}\|^{2} \\
=-2 \operatorname{Im}\left(u_{\varepsilon} \delta z, \tilde{\gamma}\right)-2 \operatorname{Im}(\tilde{\gamma} z, \tilde{\gamma})-2\left(\varepsilon W^{2}, \delta\right)+2\left(|u|^{2}-\left|u_{\varepsilon}\right|^{2}, \tilde{\rho}\right) \\
\quad+2[-A+\sigma(\mu-\sigma)](\delta, \tilde{\rho})-2 \varepsilon(\sigma-\mu)\left(W^{2}, \tilde{\rho}\right) .
\end{aligned}
$$

In what follows, we will estimate (4.6) by term by term. By the definition of the linear operator $A$, we have

$$
\begin{aligned}
-(A \delta, \tilde{\rho}) & =\sum_{k=1}^{n}\left(A_{k} \delta, \tilde{\rho}\right)=\sum_{k=1}^{n}\left(B_{k}^{*} B_{k} \delta, \tilde{\rho}\right)=\sum_{k=1}^{n}\left(B_{k} \delta, B_{k} \tilde{\rho}\right) \\
& \leq \frac{1}{2} \sum_{k=1}^{n}\left(\left\|B_{k} \delta\right\|^{2}+\left\|B_{k} \tilde{\rho}\right\|^{2}\right) \leq \frac{n C^{2}}{2}\left(\|\delta\|^{2}+\|\tilde{\rho}\|^{2}\right)
\end{aligned}
$$

Using the Young inequality, we have

$$
\begin{aligned}
& -2 \operatorname{Im}\left(u_{\varepsilon} \delta z, \tilde{\gamma}\right) \leq \frac{2 \alpha-1}{2}\|\tilde{\gamma}\|^{2}+\frac{8}{2 \alpha-1}\|\delta\|^{2}\left\|u_{\varepsilon}\right\|^{2}\|z\|^{2}, \\
& -2 \operatorname{Im}(\tilde{\gamma} z, \tilde{\gamma}) \leq \frac{2 \alpha-1}{2}\|\tilde{\gamma}\|^{2}\|z\|^{2}, \\
& -2\left(\varepsilon W^{2}, \delta\right) \leq \sigma\|\delta\|^{2}+\frac{4}{\sigma} \varepsilon^{2}\left\|W^{2}\right\|^{2}, \\
& 2\left(|u|^{2}-\left|u_{\varepsilon}\right|^{2}, \tilde{\rho}\right) \leq 2\left|\left\|u_{\varepsilon}\right\|^{2}-\|u\|^{2}\right|\|\tilde{\rho}\|^{2}, \\
& -2 \sigma(\mu-\sigma)(\delta, \tilde{\rho}) \leq \frac{\mu-\sigma}{2}\|\tilde{\rho}\|^{2}+4(\mu-\sigma)\|\delta\|^{2}, \\
& -2 \varepsilon(\sigma-\mu)\left(W^{2}, \tilde{\rho}\right) \leq \frac{\mu-\sigma}{2}\|\tilde{\rho}\|^{2}+4 \varepsilon^{2}(\mu-\rho)\left\|W^{2}\right\|^{2} .
\end{aligned}
$$

Therefore, by the above inequalities and Lemma 3.1, we obtain

$$
\frac{d}{d t}\left(\|\tilde{\gamma}\|^{2}+\|\delta\|^{2}+\|\tilde{\rho}\|^{2}\right)+C_{2}\left(\|\tilde{\gamma}\|^{2}+\|\delta\|^{2}+\|\tilde{\rho}\|^{2}\right) \leq C_{3} \varepsilon^{2}\left\|W^{2}\right\|^{2}
$$

where $C_{2}, C_{3}$ are constants depending on $\alpha, \sigma, \mu, n, C$, and $\left\|u_{0}\right\|^{2}$.

Applying the Gronwall lemma to (4.7), we get

$$
\|\tilde{\gamma}\|^{2}+\|\delta\|^{2}+\|\tilde{\rho}\|^{2} \leq e^{-C_{2} t}\left(\left\|\tilde{\gamma}_{0}\right\|^{2}+\left\|\delta_{0}\right\|^{2}+\left\|\tilde{\rho}_{0}\right\|^{2}\right)+C_{3} \varepsilon^{2}\left\|W^{2}\right\|^{2}
$$

Note that $\tilde{\gamma}_{0}=0, \delta_{0}=0$, and $\tilde{\rho}_{0}=0$, so, by $(4.8)$, we have

$$
\lim _{\varepsilon \rightarrow 0}\left(\|\tilde{\gamma}(t)\|^{2}+\|\delta(t)\|^{2}+\|\tilde{\rho}(t)\|^{2}\right)=0, \quad \text { for all } t>0
$$

which implies that

$$
\lim _{\varepsilon \rightarrow 0} \gamma(t)=0 \quad \text { and } \quad \lim _{\varepsilon \rightarrow 0} \delta(t)=0, \quad \text { for all } t>0 .
$$

This completes the proof. 
Theorem 4.1 Assume that $\left\{A_{\varepsilon}(\omega)\right\}_{\varepsilon \in(0,1)}$ are attractors for system (1.1). Then, for $\mathbb{P}$-almost every $\omega \in \Omega$,

$$
\lim _{\varepsilon \rightarrow 0} \operatorname{dist}\left(A_{\varepsilon}(\omega), A_{0}\right)=0
$$

\section{Competing interests}

The authors declare that they have no competing interests.

\section{Authors' contributions}

All authors contributed equally to the writing of this paper. All authors read and approved the final manuscript.

\section{Author details}

'School of Mathematics and Information Sciences, North China University of Water Resources and Electric Power, Zhengkai Road, Zhengzhou, 450011, P.R. China. ${ }^{2}$ School of Mathematics and Information Science, Henan Polytechnic University, Jiaozuo Road, Jiaozuo, 454150, P.R. China.

\section{Acknowledgements}

The authors express their sincere thanks to the anonymous referees for a very careful reading and for providing many valuable comments and suggestions, which led to an improvement of this paper. The first author is supported by the Fundamental Research Foundation for PHD of NCWU (No. 40291).

\section{Received: 4 October 2014 Accepted: 17 November 2014 Published: 01 Dec 2014}

\section{References}

1. Fukuda, I, Tsutsumi, M: On the coupled Klein-Gordon-Schrödinger equations, III. Math. Jpn. 24, 307-321 (1979)

2. Guo, B, Li, Y: Attractor for dissipative Klein-Gordon-Schrödinger equations in R3 . J. Differ. Equ. 136, 356-377 (1997)

3. Wang, BX, Lange, H: Attractors for the Klein-Gordon-Schrödinger equation. J. Math. Phys. 40(5), 2445-2457 (1999)

4. Lu, KN, Wang, BX: Global attractors for the Klein-Gordon-Schrödinger equations in unbounded domains. J. Differ. Equ. $170,281-361$ (2001)

5. Biler, P: Attractors for the system of Schrödinger and Klein-Gordon equations with Yukawa coupling. SIAM J. Math. Anal. 21(5), 1190-1212 (1990)

6. Zhao, CD, Zhou, SF: Compact kernel sections for nonautonomous Klein-Gordon-Schrödinger equations on infinite lattices. J. Math. Anal. Appl. 332, 32-56 (2007)

7. Lu, KN, Wang, BX: Upper semicontinuity of attractors for the Klein-Gordon-Schrödinger equation. Int. J. Bifurc. Chaos Appl. Sci. Eng. 15(1), 157-168 (2005)

8. Temam, R: Infinite Dimensional Dynamical Systems in Mechanics and Physics. Springer, New York (1995)

9. Bates, PW, Lu, KN, Wang, BX: Attractors for lattice dynamical systems. Int. J. Bifurc. Chaos Appl. Sci. Eng. 11(1), 143-153 (2001)

10. Chow, SN: Lattice dynamical systems. In: Dynamical Systems. Lect. Notes in Math., vol. 1822, pp. 1-102 (2003)

11. Chow, SN, Paret, JM: Pattern formation and spatial chaos in lattice dynamical systems. IEEE Trans. Circuits Syst. 42, 746-751 (1995)

12. Fan, XM, Wang, YG: Attractors for a second order nonautonomous lattice dynamical system with nonlinear damping. Phys. Lett. A 365, 17-27 (2007)

13. Wang, BX: Attractors for reaction-diffusion equations in unbounded domains. Physica D 128, 41-52 (1999)

14. Wang, BX: Dynamics of lattice systems on infinite lattices. J. Differ. Equ. 221, 224-245 (2006)

15. Abdallah, AY: Long-time behavior for second order lattice dynamical systems. Acta Appl. Math. (2008). doi:10.1007/s10440-008-9281-8

16. Abdallah, AY: Asymptotic behavior of the Klein-Gordon-Schrödinger lattice dynamical systems. Commun. Pure Appl. Anal. 5(1), 55-69 (2006)

17. Zhou, SF: Attractors for first order dissipative lattice dynamical systems. Physica D 178, 51-61 (2003)

18. Bates, PW, Chen, X, Chmaj, A: Travelling waves of bistable dynamics on a lattice. SIAM J. Math. Anal. 35, 520-546 (2003)

19. Chua, LO, Roska, T: The CNN paradigm. IEEE Trans. Circuits Syst. 40, 147-156 (1993)

20. Kapval, R: Discrete models for chemically reacting systems. J. Math. Chem. 6, 113-163 (1991)

21. Chow, SN, Paret, JM, Vleck, ES: Pattern formation and spatial chaos in spatially discrete evolution equations. Random Comput. Dyn. 4, 109-178 (1996)

22. Carrol, TL, Pecora, LM: Synchronization in chaotic systems. Phys. Rev. Lett. 64, 821-824 (1990)

23. Fabiny, L, Colet, P: Coherence and phase dynamics of spatially coupled solid-state lasers. Phys. Rev. A 47, 4287-4296 (1993)

24. Bates, PW, Lisei, H, Lu, KN: Attractors for stochastic lattice dynamical systems. Stoch. Dyn. 6, 1-21 (2006)

25. LV, Y, Sun, J: Dynamical behavior for stochastic lattice systems. Chaos Solitons Fractals 27, 1080-1090 (2006)

26. LV, Y, Sun, J: Asymptotic behavior of stochastic discrete complex Ginzburg-Landau equations. Physica D 221, 157-169 (2006)

27. Huang, JH: The random attractor of stochastic Fitzburg-Nagumo equations in an infinite lattice with white noises. Physica D 233, 83-94 (2007)

28. Ruelle, D: Characteristic exponents for a viscous fluid subjected to time dependent forces. Commun. Math. Phys. 93 , 285-300 (1984) 
29. Arnold, L: Random Dynamical Systems. Springer, Berlin (1998)

30. Crauel, H, Debussche, A, Flandoli, F: Random attractors. J. Dyn. Differ. Equ. 9, 307-341 (1997)

31. Yan, WP, Ji, SG, Li, Y: Random attractors for stochastic discrete Klein-Gordon-Schrödinger equations. Phys. Lett. A 373 , 1268-1275 (2009)

32. Da Prato, GD, Zabczyk, J: Ergodicity for Infinite Dimensional Systems. London Mathematical Society Lecture Note Series, vol. 229. Cambridge University Press, Cambridge (1996)

33. Caraballo, T, Langa, JA, Robinson, C: Upper semicontinuity of attractors for small random perturbations of dynamical systems. Commun. Partial Differ. Equ. 23, 1557-1581 (1998)

10.1186/1687-1847-2014-300

Cite this article as: Li and Sun: Upper semicontinuity of attractors for small perturbations of

Klein-Gordon-Schrödinger lattice system. Advances in Difference Equations 2014, 2014:300

Submit your manuscript to a SpringerOpen ${ }^{\circ}$ journal and benefit from:

- Convenient online submission

- Rigorous peer review

- Immediate publication on acceptance

- Open access: articles freely available online

- High visibility within the field

- Retaining the copyright to your article

Submit your next manuscript at $>$ springeropen.com 